\title{
Comparison of activity levels measured by a wrist worn accelerometer and direct observation in young children
}

\author{
Kurosh Djafarian $^{1,2,3}$, John R. Speakman ${ }^{1,2}$, Joanne Stewart ${ }^{1,2}$, Diane M. Jackson ${ }^{1,2}$ \\ ${ }^{1}$ Department of Clinical Nutrition, School of Nutritional Sciences and Dietetics, Tehran University of Medical Sciences, Tehran, \\ Iran \\ ${ }^{2}$ The Division of Obesity and Metabolic Health, Rowett Institute of Nutrition and Health, University of Aberdeen, Aberdeen, UK \\ ${ }^{3}$ The Institute of Biological and Environmental Sciences, University of Aberdeen, Aberdeen, UK \\ Email: d.jackson@abdn.ac.uk
}

Received 15 November 2013; revised 10 December 2013; accepted 17 December 2013

Copyright (C) 2013 Kurosh Djafarian et al. This is an open access article distributed under the Creative Commons Attribution License, which permits unrestricted use, distribution, and reproduction in any medium, provided the original work is properly cited.

\begin{abstract}
Introduction: Motion sensors are mechanical and electronic devices, which detect the body movement and provide an estimate of physical activity in children and adults. However, they need to be validated against criterion methods such as direct observation. The purpose of this study was to validate a wrist worn accelerometer to quantify the physical activity of children, by comparison to direct observation using the Children's Activity Rating Scale (CARS). Materials and Methods: Data were recorded from 42 children, aged 3 - 5 years ( 22 boys and 20 girls), of whom each was observed each minute for 2 hours using Children's Activity Rating Scale (CARS) while they wore the Actiwatch. Results: The CARS score and activity counts from the accelerometer were averaged over 1- to 10-minute periods across all individuals. There was a significant positive correlation between the mean CARS scores and the mean Actiwatch counts over simultaneous 1- to 10 -minute periods ranging from $r=0.41$ to $r=0.63(P<0.001)$. To assess validity of the data, a cross validation method was applied. There was no significant difference between the predicted and the observed CARS scores in the validation sample. Given the data from the Actiwatch (averaged over a 5-minute epoch), the equivalent CARS score could be calculated with a 95\% confidence level of plus or minus 0.74 CARS units. Conclusion: These data suggest that the Actiwatch (a wrist worn accelerometer) is a valid tool for assessing levels of physical activity in young children.
\end{abstract}

Keywords: Accelerometer; Physical Activity; Children; Observation

\section{INTRODUCTION}

The prevalence of obesity among children in both developed and developing countries has reached epidemic proportions [1]. Attention has been directed to understand the causes, consequences, treatment, and prevention of this current epidemic. Change in children's dietary and physical activity may play a central role in the obesity epidemic [2]. There is little evidence to suggest that children have increased their consumption of energy in recent years $[3,4]$, consequently attention has shifted towards reductions in the levels of physical activity as a major potential contributor to this epidemic. Although, the role of physical activity in the development of obesity is less clear, there are some studies that show a positive relationship between childhood obesity and physical inactivity $[5,6]$. However, a debated issue remains.

Epidemiological studies indicate a decrease in physiccal activity among children and adolescents during last decades [7]. However, most epidemiological research is based on subjective indirect measures of physical activity, such as activity questionnaires, which rely on the subject's capacity to recall past activity patterns. Such recall is prone to error, and this is perhaps compounded for young children where the recalled activity is made second hand by a parent or caregiver. To obtain greater insight into the interaction between physical activity and childhood obesity, there is a need for more objective and reliable measures of physical activity in free-living conditions [8].

A number of methods for assessing physical activity in children are available, each with advantages and disadvantages. Perhaps the most reliable measure of physical activity is direct observation. This method, however, has several drawbacks. It is labour intensive, and the presence of the observer or a camera may disrupt natural 
behaviour patterns. Self-report questionnaires and diaries overcome the problems associated with direct observation, but while they are less invasive, they are also prone to reporting errors [9]. Measurements of energy expenditure levels, either using Doubly Labelled Water or by heart rate monitoring provide measures of energy expended on activity that can be collected remotely without the need for persistent direct observation. But these methods are invasive and expensive and hence unsuited to large-scale field measurements. Accelerometers provide a method that potentially overcomes many of the disadvantages of other techniques [10,11]. Modern accelerometers are compact and lightweight and many can be worn on the wrist like a watch, making them very unobtrusive. However, these devices generally generate information on levels of physical activity in terms of "counts". How this measure translates into categories of activity used in direct observation is uncertain and is likely to vary with the different commercially available loggers and precise details of how they are used.

Some commercially available accelerometers have been validated and calibrated in populations of interest $[12,13]$. The location of attachment on the body may affect the accuracy of these devices. One study found that different correlation between energy expenditure on the treadmill and activity counts was collected with an accelerometer placed on different locations of body. Attaching the accelerometer on ankle, hip and wrist showed most accurate prediction of energy expenditure [14]. Similar finding has been observed in a recent study in children and adults [15]. Wrist-worn accelerometers have been shown to accurately measure activities that are underestimated by waist-mounted monitors, such as cycling and rowing, but may overestimate those activities that require rapid hand movements like typing [16]. Despite some limitations, accelerometers have been found to give useful objective measurements of physical activity in young children [17]. Among them, the Actiwatch is small, more convenient to use and with a large capacity for monitoring and storing physical activity data, and is easy for the children to wear over protracted periods of time. This wrist-worn accelerometer has never been validated under free-living conditions and activity counts cannot be translated readily into activity intensities. Therefore, the evaluation of this small accelerometer to detect physical activity in children may help future studies investigate the association between children's physical activity and health.

The aims of this study were to:

Firstly, compare a wrist worn accelerometer (the Actiwatch) with direct observation using the Children's Activity Rating Scale (CARS) and to produce a formula, which will calculate the intensity of activities based on Actiwatch counts. And secondly, compare the relationship using observation periods and epochs greater than 1 minute.

\section{METHODS}

\subsection{Subjects}

Participants for the study were 42 Caucasian children (22 boys, 20 girls), with a mean age of $4.06 \pm 0.76$ years. The Grampian Research Ethics Committee approved the study protocol and subjects were recruited from local nursery schools around Aberdeen, NE Scotland, UK. Written informed consent was obtained from the child's parents or guardian and verbal consent from the child.

\subsection{Observations}

Prior to the placement of the Actiwatch, the weight of children dressed in light clothing was recorded $(0.1 \mathrm{~kg})$ using high precision electronic scales (SECA, Vogel \& Halke, Hamburg, Germany), while height was measured up to $0.1 \mathrm{~cm}$ using a portable stadiometer (Leicester stadiometer, Child growth Foundation, London, UK). These measurements were carried out at the nurseries by researchers. The children were then allowed to take part in their normal nursery routine. Nurseries in the UK generally consist of social groups of similar aged children along with a number of care workers. They engage in a number of structured activities, such as meals and organised play activities, as well as free time when their activity is unstructured both inside and outside the nurseries. After 5 min acclimation time, observations were made of individual children for a period of approximately 2 hours during which time they engaged in both structured and unstructured activity.

\subsection{Physical Activity Monitor (Actiwatch)}

The Actiwatch-L (Cambridge Neurotechnology Ltd.) is a small accelerometer containing a miniature uniaxial recorder, which produces a signal as the unit is accelerated over $0.05 \mathrm{~g}$. This signal is measured 32 times per second and processed to provide the digital integration of the amount and duration of movement. The monitor stores this information in non-volatile memory as activity counts. These activity counts can be downloaded and analysed using the Actiwatch analysis software. The Actiwatch has filters to exclude readings outside the 3 - 11 $\mathrm{Hz}$ ranges. For this study, the Actiwatch was worn on the non-dominant wrist of each subject and it was set up to store total activity counts per minute (epoch) during the observation period.

\subsection{Children's Activity Rating Scale}

Children's Activity Rating Scale (CARS) was used to categorise the intensity of physical activities and discriminate between different levels of activities in sub- 
jects. In this study, there were two observers and CARS scores were measured each minute by them. Each observer scored only one child and continuous monitoring was limited up to 2 hours. The CARS includes a wide variety of physical activities and exercise intensities, which can be classified to 5 categories according to a rating system developed by Puhl et al. [18]. The level 1 activities basically represent very little body movement or sedentary activity. The level 2 activities involve movement of a limb (arm or leg) or trunk without movement of the whole body weight from one place to another. Level 3, 4 and 5 activities are labelled as moving the entire body weight from one location to another slowly (level 3), moderately (level 4) or fast or very fast (level 5). The CARS score was recorded by averaging all levels of physical activity (and inactivity) within each minute. Actiwatch can be programmed to record PA epochs of less than one-minute, however, the shortest measurement period with CARS is one minute, hence this was chosen.

\subsection{Statistical Analysis}

Descriptive statistics were carried out for all variables including CARS scores and Actiwatch output (counts). Activity counts for 1 to 10 minute time intervals (Epochs) were categorised and matched with average of accompanying 1 to 10 minutes' CARS scores for each subject's data. A correlation coefficient was computed to quantify the linear relationship between CARS scores and Actiwatch counts for those different time intervals. Linear regression analysis was also applied with curve estimation, between the Actiwatch counts and the CARS scores. Multiple-linear regression was used to identify effects of other variables. Statistical Software of MINITAB (Version 14) was used for all statistical analyses. The level of statistical significance was set as $\mathrm{P}<0.05$.

\section{RESULTS}

Forty-two children ( 3 - 5 years) took part in the study. Subject characteristics are given in Table 1. There was no difference between boys or girls in average Actiwatch counts or CARS scores.

The average duration of direct observation was 109.21 \pm 12.24 minutes. Figure 1 shows the trend of correlation between Actiwatch counts for 1 to 10 minute intervals and corresponded CARS scores (Ranging from $\mathrm{r}=0.41$ to $\mathrm{r}=0.63, \mathrm{P}<0.001)$.

To consider the accuracy and resolution of correlation, among different intervals, 5 minutes (Figure 2) was chosen for the subsequence of analysis $(\mathrm{r}=0.59, \mathrm{P}<0.01)$. However, it was found that some individual data did not correlate. On closer inspection of the data it was found that these subjects did not have a wide range of activities $(\mathrm{n}=11)$, and when these subject were excluded the relationship was stronger $(\mathrm{r}=0.67, \mathrm{P}<0.001)$.

To find out whether the results found are valid a cross validation method was applied [19]. In this method the subjects were randomly divided in two groups of a validation $(\mathrm{n}=21)$ and a cross-validation sample $(\mathrm{n}=21)$. On the cross-validation sample, the correlation between CARS score and Actiwatch counts was significant $(\mathrm{r}=$ $0.52, \mathrm{P}<0.001$ ). The regression equation (CARS levels $=1.65+0.000432 \times$ Actiwatch counts) to predict CARS levels by Actiwatch counts did not intercept at zero. Therefore, the log of Actiwatch data was used to change the intercept and have linear relationship (CARS levels = $-0.138+0.758 \log$ Actiwatch counts). When the equation developed from cross validation sample was applied to the data from the validation sample; there was no significant difference between the predicted and the observed CARS levels and the mean difference between measured and predicted CARS scores was $0.025( \pm 0.38)$ (Figure 3).

Table 2 shows the CARS accuracy based on different samples of Actiwatch (5 minutes epochs) with confidence level of $95 \%$. Given the data from the Actiwatch (averaged over a 5-minute epoch) the equivalent CARS score could be calculated with a $95 \%$ confidence level of plus or minus 0.74 CARS units.

Table 1. Subject characteristics.

\begin{tabular}{cccccccc}
\hline & \multicolumn{2}{c}{ Boys (22) } & \multicolumn{2}{c}{ Girls (20) } & \multicolumn{2}{c}{ Total (42) } \\
\cline { 2 - 7 } & Mean & SD & Mean & SD & Mean & SD \\
\hline Age (years) & 4.26 & 0.73 & 3.84 & 0.75 & 4.06 & 0.76 \\
Height (kg) & 115.15 & 22.79 & 104.32 & 5.74 & 110.13 & 17.82 \\
Weight (cm) & 18.78 & 2.90 & 18.13 & 2.49 & 18.48 & 2.70 \\
Actiwatch (Counts) & 814.44 & 271.35 & 774.68 & 329.78 & 795.51 & 297.51 \\
CARS & 1.99 & 0.17 & 1.99 & 0.23 & 1.99 & 0.20 \\
\hline
\end{tabular}

Table 2. The CARS accuracy based on different samples of Actiwatch (5 minutes epochs).

\begin{tabular}{cc}
\hline Samples of Actiwatch (5 min epoch) & Confidence interval \\
\hline 1 & 0.74 \\
10 & 0.24 \\
20 & 0.17 \\
40 & 0.12 \\
60 & 0.10 \\
80 & 0.08 \\
100 & 0.07 \\
\hline
\end{tabular}




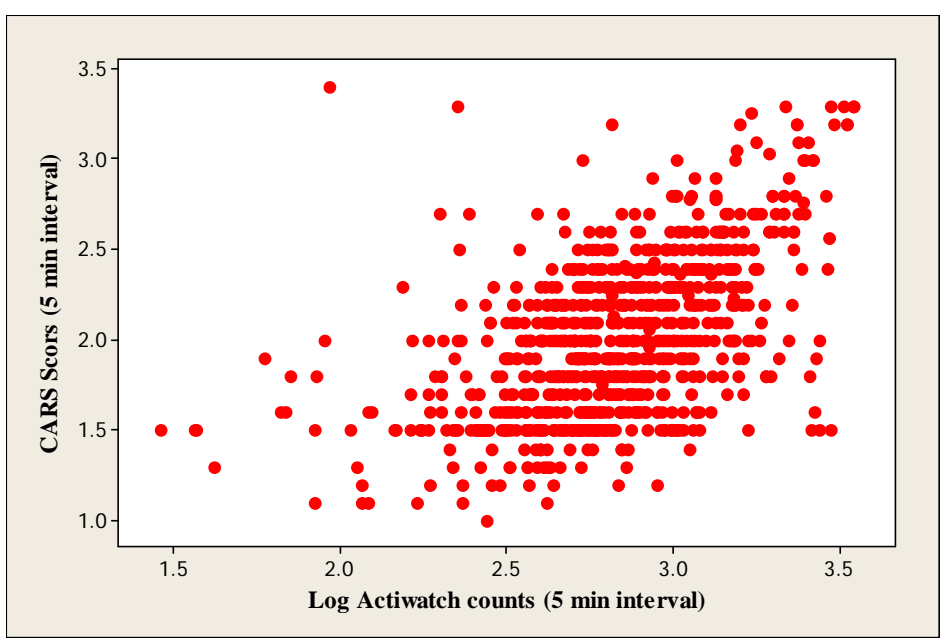

Figure 1. Trend of correlation between Actiwatch counts and corresponding CARS scores.

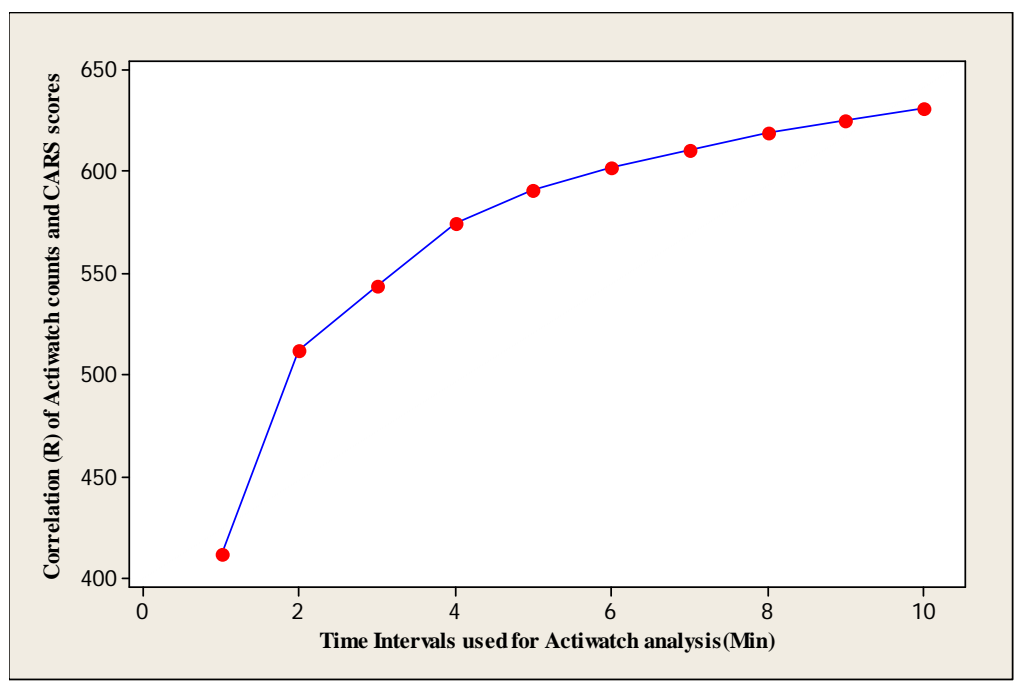

Figure 2. Scoter plot of correlation between CARS scores (5 minutes interval) and corresponding log Actiwatch counts.

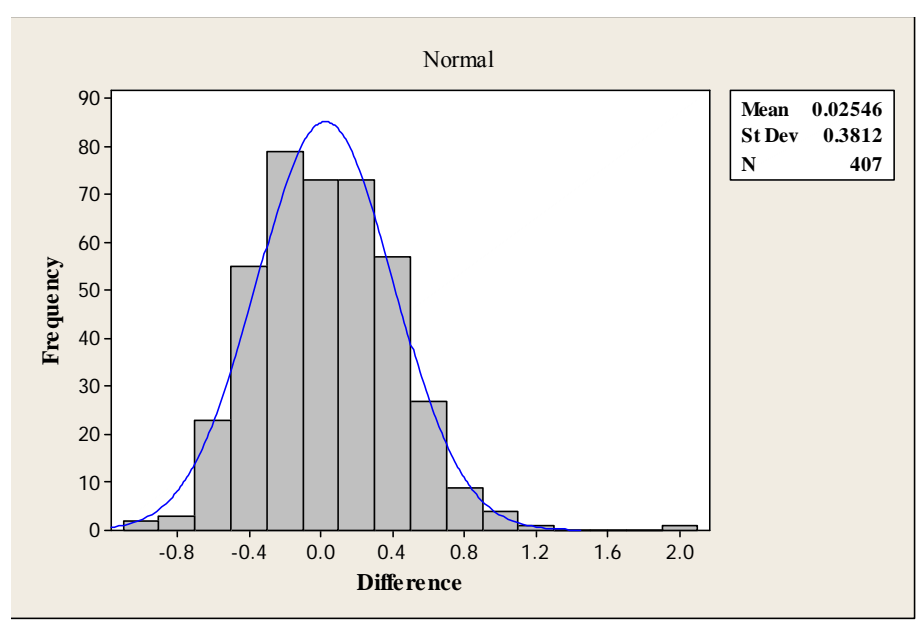

Figure 3. Histogram of difference between logs of predicted and observed CARS levels. 


\section{DISCUSSION}

There is increasing interest in the health benefits of physical activity in children. However, practical and accurate means to quantify this complex behavior are required. Accelerometry monitors are widely used to assess physical activity due to their ease of use over long periods, and provision of detailed information about the intensity, frequency and duration of physical activity.

This study assessed the validity of the Actiwatch in quantifying physical activity in children. Our findings are consistent with the results of similar studies in children $[8,13,17]$. These results demonstrated that movement measured using uniaxial accelerometers could be used to accurately measure various types and intensity of activities in children.

A study by Finn and Specker that used CARS as the criterion measure of activity, reported that the Actiwatch (Mini Mitter) attached to the waist provides an accurate assessment of children physical activity in free-living condition particularly in those who had been more active. The within-childcorrelation between 3-minute Actiwatch counts and CARS scores was 0.74 [13]. In the current study the Actiwatch was placed on wrist of subjects and validated against CARS, which has never been done before. However, it was argued that the placement of activity monitor might affect the outcome of measurement [10]. Therefore, the monitor placement might be a third factor, which needs to be considered when comparing different accelerometer studies.

The different time intervals seem to be challenging issue when assessing physical activity with an accelerometer and it should be considered. Using 1-minute interval in children might limit the accuracy of measuring activity and cause misclassification of intensity activity levels [20]. To our knowledge, only one study has investigated the effect of different time sampling epochs on capturing physical intensity in children [20]. This study has observed a significant effect of time sampling epochs for time spent at very high and high intensity activities. Although, this study has shown that using shorter epochs give a more detailed picture of PA in children, the accelerometer (CSA Activity monitor), which was used in this study was validated in defined condition for one min interval not less [21]. Therefore, further investigations are required to determine the optimum epoch length for assessing PA intensity in children. Testing epochs less than 1 minute was beyond the scope of this study as the CARS uses one minute observations [18]. In current study, 10 different time epochs (1 - 10 minutes) have been used. Increasing time intervals caused the relationship to become stronger (Figure 1); however, the trend of correlation was nearly flat after 5-min interval, therefore, 5-min epoch was chosen to have optimum accuracy and resolution for this study.
In order to test the validity of the developed equation for CARS prediction a cross-validation was performed. No significant difference between measured and predicted CARS scores was observed in the validation sample. It should be considered that we produced the equation from children with limited variation in their activities. Therefore, it must be noted that prediction of CARS scores from Actiwatch counts should not be performed outside the range of activity monitor data found in the present study (Figure 2).

These data indicate that the Actiwatch activity monitor seems to be a valid tool for assessing physical activity performed by children age 2 - 5 years. Due to wide variety of activity levels in defined conditions like laboratory (e.g. treadmill), the validation studies have shown strong correlations between activity counts and criterion methods and vice versa in field condition (e.g. free living condition [14,17]. The Actiwatch monitor, a uniaxial accelerometer, used in this study, is small, wrist-worn device, easy to use, well tolerated by children and allows them to engage in normal daily activities. Data from some early investigations indicate that uniaxial accelerometers may not be able to detect physical activity as effective as triaxial accelerometers [22]. However, a number of studies consistent with this study have found that the uniaxial accelerometers provide valid data and they are equally effective as triaxial models in qualifying physical activity and estimating EE in children and adolescents $[10,13,23]$. Therefore, the relative small size of this wrist worn Actiwatch along with its validity and precision provide a practical tool for assessing physical activity particularly for younger children.

\section{CONCLUSION}

A limitation of our study was the time that the children spent performing vigorous activities, which was too short. In addition, the CARS is not able to measure PA for less than one-minute interval and therefore we may be missing some vigorous activities [20]. In summary, this study reveals that this Actiwatch monitor is a valid means for the assessment of physical activity in children. In addition, an equation for prediction of CARS scores to use in other studies was developed and cross-validated. Further studies particularly with measuring EE as criterion measure might be beneficial to confirm these data.

\section{ACKNOWLEDGEMENTS}

This work was granted by The Grampian Research Ethics Committee. We acknowledge the contribution of the children to the present study. We also declare that we have no conflict of interest.

\section{REFERENCES}

[1] Raychaudhuri, M. and Sanyal, D. (2012) Childhood obe- 
sity: Determinants, evaluation, and prevention. Indian Journal of Endocrinology and Metabolism, 16, S192-S194.

[2] Patrick, K., Norman, G.J., Calfas, K.J., Sallis, J.F., Zabinski, M.F., Rupp, J. and Cella, J. (2004) Diet, physical activity, and sedentary behaviors as risk factors for overweight in adolescence. Archives of Pediatrics and Adolescent Medicine, 158, 385-390.

http://dx.doi.org/10.1001/archpedi.158.4.385

[3] Troiano, R.P. and Flegal, K.M. (1998) Overweight children and adolescents: Description, epidemiology, and demographics. Pediatrics, 101, 497-504.

[4] Reilly, J.J. and Dorosty, A.R. (1999) Epidemic of obesity in UK children. Lancet, 27, 1874-1875. http://dx.doi.org/10.1016/S0140-6736(99)04555-9

[5] Gortmaker, S.L., Must, A., Sobol, A.M., Peterson, K., Colditz, G.A. and Dietz, W.H. (1996) Television viewing as a cause of increasing obesity among children in the united states, 1986-1990. Archives of Pediatrics and Adolescent Medicine, 150, 356-362.

http://dx.doi.org/10.1001/archpedi.1996.02170290022003

[6] Saris, W.H., Blair, S.N., van Baak, M.A., Eaton, S.B., Davies, P.S., Di Pietro L., et al. (2003) How much physical activity is enough to prevent unhealthy weight gain? Outcome of the IASO 1st Stock Conference and consensus statement. Obesity Reviews, 4, 101-114. http://dx.doi.org/10.1046/j.1467-789X.2003.00101.x

[7] Livingstone, M.B.E., Robson, P.J., Wallace, J.M.W. and McKinley, M.C. (2003) How active are we? Levels of routine physical activity in children and adults. Proceedings of the Nutrition Society, 62, 681-701. http://dx.doi.org/10.1079/PNS2003291

[8] Klesges, L.M. and Klesges, R.C. (1987) The assessment of children's physical-activity-A comparison of methods. Medicine \& Science in Sports \& Exercise, 19, 511517.

http://dx.doi.org/10.1249/00005768-198710000-00015

[9] Going, S.B., Levin, S., Harrell, J., Stewart, D., Kushi, L., Cornell, C.E., et al. (1999) Physical activity assessment in American Indian schoolchildren in the Pathways study. American Journal of Clinical Nutrition, 69, 788S-795S.

[10] Welk, G.J., Corbin, C.B. and Dale, D. (2000) Measurement issues in the assessment of physical activity in children. Research Quarterly for Exercise \& Sport, 71, S59S73.

[11] Jackson, D.M., Reilly, J.J., Kelly, L.A., Montgomery, C., Grant, S. and Paton, J.Y. (2003) Objectively measured physical activity in a representative sample of 3- to 4year-old children. Obesity Research, 11, 420-425. http://dx.doi.org/10.1038/oby.2003.57

[12] Welk, G.J. and Corbin, C.B. (1995) The validity of the Tritrac-R3d activity monitor for the assessment of physical activity in children. Research Quarterly for Exercise and Sport, 66, 202-209.

http://dx.doi.org/10.1080/02701367.1995.10608834

[13] Finn, K.J. and Specker, B. (2000) Comparison of actiwatch $(\mathrm{R})$ activity monitor and children's activity rating scale in children. Medicine \& Science in Sports \& Exercise, 32, 1794-1797.

http://dx.doi.org/10.1097/00005768-200010000-00021

[14] Melanson, E.L. and Freedson, P.S. (1995) Validity of the computer-science and applications, Inc. (Csa) activity monitor. Medicine \& Science in Sports \& Exercise, 27, 934-940.

http://dx.doi.org/10.1249/00005768-199506000-00021

[15] Heil, D.P. (2006) Predicting activity energy expenditure using the actical (R) activity monitor. Research Quarterly for Exercise \& Sport, 77, 64-80.

[16] Sugimoto, A., Hara, Y., Findley, T.W. and Yonemoto, K. (1997) A useful method for measuring daily physical activity by a three-direction monitor. Scandinavian Journal of Rehabilitation Medicine, 29, 37-42.

[17] Puyau, M.R., Adolph, A.L., Vohra, F.A. and Butte, N.F. (2002) Validation and calibration of physical activity monitors in children. Obesity Research, 10, 150-157. http://dx.doi.org/10.1038/oby.2002.24

[18] Puhl, J., Greaves, K., Hoyt, M. and Baranowski, T. (1990) Children's activity rating-scale (Cars)-Description and calibration. Research Quarterly for Exercise and Sport, 61, 26-36. http://dx.doi.org/10.1080/02701367.1990.10607475

[19] Reilly, J.J., Coyl, J., Kelly, L., Burke, G., Grant, S. and Paton, J.Y. (2003) An objective method for measurement of sedentary behavior in 3- to 4-year olds. Obesity Research, 11, 1155-1158.

http://dx.doi.org/10.1038/oby.2003.158

[20] Nilsson, A., Ekelund, U., Yngve, A. and Sjostrom, M. (2002) Assessing physical activity among children with accelerometers using different time sampling intervals and placements. Pediatric Exercise Science, 14, 87-96.

[21] Eston, R.G., Rowlands, A.V. and Ingledew, D.K. (1998) Validity of heart rate, pedometry, and accelerometry for predicting the energy cost of children's activities. Journal of Applied Physiology, 84, 362-371.

[22] Freedson, P.S. and Miller, K. (2000) Objective monitoring of physical activity using motion sensors and heart rate. Research Quarterly for Exercise \& Sport, 71, S21S29.

[23] Bray, M.S., Wong, W.W., Morrow, J.R., Butte, N.F. and Pivarnik, J.M. (1994) Caltrac versus calorimeter determination of 24-H energy expenditure in female children and adolescents. Medicine and Science in Sports and EXercise, 26, 1524-1530.

http://dx.doi.org/10.1249/00005768-199412000-00017 